\title{
Using a Mixed Methods Approach to Examine the Effectiveness of Inpatient Rehabilitation Services Following a Programme of Planned Bed Closures
}

\author{
Gabriella J. Mutale $\cdot$ Claire de Motte $\cdot$ Di Bailey
}

Received: 6 November 2019/Accepted: 29 February 2020/Published online: 12 March 2020

(C) The Author(s) 2020

\begin{abstract}
This study examined inpatient rehabilitations service in one area in England following a programme of planned bed closures in parts of the service. The study examined changes to admission rates and length of stay in the parts of the service that existed prior to and after the bed closure programme. A mixed method evaluation design was used. Quantitative data relating to admissions and length of inpatients stays bed days was compared for the 3 years up to the bed closures and for the 3 years afterwards. Similarly use of mental health sections for service users being admitted, and contacts with community mental health teams were also compared for the same 3 -year pre and post period. Qualitative data from service users, carers of service users, and staff were analyzed thematically to provide an insight into any changes in rehabilitation service usage as a result of the closure programme. In the rehabilitation services that existed before and after bed closures in other parts of the service quantitative findings showed a significant reduction post-rehabilitation stay in all measures except contact with community teams. Qualitative analysis revealed that this is because rehabilitation enables a successful transition into the community and allows for effective relationships to be built between staff and service user. The reduction seen in service
\end{abstract}

G. J. Mutale · C. de Motte $(\bowtie) \cdot$ D. Bailey

School of Social Sciences, Nottingham Trent University, Shakespeare Street, Nottingham NG1 4FQ, UK

e-mail: claire.demotte@ntu.ac.uk utilization suggests rehabilitation has the potential to reduce the revolving door to inpatient metal health care.

Keywords Inpatient mental health rehabilitation . Severe and enduring mental illness - Recovery · Evaluation research

\section{Introduction}

Mental health rehabilitation plays an important role in the recovery for people with complex mental health needs "which maximizes an individual's quality of life and social inclusion by encouraging interpersonal skills and promoting independence and autonomy. This provides service users with hope for the future and the ambition to achieve successful community living through appropriate support" [1], (p. 163). In England, mental health rehabilitation services typically treat service users with complex needs including severe and enduring mental illness, challenging behavior, and poor social function [2]. Figures estimate that $20 \%$ of service users with a new diagnosis will require support from a rehabilitation service during some point of their mental health recovery, and thus there is a substantial demand on rehabilitative support within England's mental health service [3]. The National Health Service (NHS) provides publicly funded inpatient rehabilitation support to service users 
when they are not well enough to leave an acute mental health ward and return to the community, but are unlikely to benefit from further care in a setting which is potentially restrictive and isolating $[4,5]$.

Modern approaches to mental health rehabilitation are delivered in a manner consistent with recoveryoriented care with the aim of enabling personal recovery in an empowering way. This places an emphasis on the individual priorities of the service user, as opposed to reducing clinical symptoms, and focuses on interventions that impact on goals and outcomes that are personally important to the service user [6].

Challenges in establishing clear outcome measures results in difficulties evidencing the effectiveness of rehabilitation and consequently specialist inpatient rehabilitation expertise that support patients in their long-term mental health recovery has been lost [7]. Understanding the outcomes of rehabilitation is a complex process, yet research does suggest there are some positive outcomes associated with rehabilitation. For example, Killaspy and Zis [8] found 5 years following discharge from inpatient rehabilitation services, $40 \%$ of their sample had progressed, $27 \%$ had remained stable and 33\% of had relapsed. High levels of service user satisfaction with the care received from rehabilitation services have been demonstrated [9] and significant improvements in Quality of Life has also been observed post discharge from inpatient rehabilitation [10]. Furthermore, it has been demonstrated that re-admissions to, and days spent in, mental health services are reduced following inpatient rehabilitation [11-14].

Despite these positive outcomes the main criticism of rehabilitation services has been advanced by the Care Quality Commission (CQC) [3] advocating that services have been providing stays which were considered too lengthy [3] and located at considerable distances to service users' homes (an average of $14 \mathrm{~km})[3,15]$.

These criticisms have contributed to a decline in the numbers of rehabilitation beds commissioned in the NHS with some Trusts undertaking a review into inpatient mental health rehabilitation bed use to inform a closure programme across Nottinghamshire, England (2013-2015). One review in the county of Nottinghamshire found that some of their rehabilitation beds were in settings that were not conducive to recovery-oriented care and the review recommended a move away from these inpatient rehabilitation services towards a greater focus and re-provision of service into the community mental health teams. This resulted in the closure of 93 inpatient beds across five units with a total of 37 inpatient rehabilitation beds remaining in two units (unit 1: high dependency; unit 2: community). Where similar changes to rehabilitation services have happened in the UK professionals have expressed their concern about whether community services can provide the specialist rehabilitation expertise needed to support service users experiencing severe and enduring mental health difficulties [7]. This debate has contributed to rehabilitation services being described as 'the forgotten need within contemporary mental health services' [4, p. 1].

This paper presents the findings from a mixedmethod evaluation study that examined the use of one high dependency and one community unit that provided inpatient rehabilitation support to service users across Nottinghamshire. These units provided a service before and after the bed closure programme in other parts of the rehab service. The Trust concerned were keen to understand whether the existing bed provision was being used to best effect and commissioned the evaluation research as an independent study to inform further service enhancement.

\section{The Study}

Changes in service use before and following an inpatient rehabilitation stay were examined using quantitative outcome measures that included: (1) the number of admissions to inpatient mental health services, (2) the number of occupied bed days in inpatient mental health services, (3) the amount of contact time with community mental health teams and, (4) the number of admissions under Mental Health Act sections. These were all examined across a 3-year time period pre and post rehabilitation stay.

Qualitative data from service users, carers, and staff were collected to understand their experiences of how inpatient rehabilitation services work towards a recovery approach from the perspective of service users and staff.

Building on previous research [11-14], it was hypothesized that inpatient rehabilitation would result in a significant change in service utilization, specifically reducing inpatient admissions to mental health services, days spent in inpatient mental health 
services, use of Mental Health Act sections, and community contact time with mental health services. Qualitative data would provide a narrative to aid understanding of the quantitative findings and any changes found.

\section{Method}

Evaluation Design

The evaluation design was guided by Pawson and Tilley's [16] realistic evaluation methodology that takes "heed of the different layers of social reality which make up and surround programmes of change" (p4). The multi-level evaluation framework [17, 18] combined levels from frameworks developed previously by Warr, Bird and Rackham [19] [context and inputs] and Kirkpatrick [20] [outcomes]. By combining these levels from both frameworks and refining these through previous research [21-23] this realistic evaluation design supported an in-depth exploration of the rehabilitation services.

\section{Setting}

In England, the NHS or the independent sector provide inpatient mental health rehabilitation. In 2018, the CQC [3] estimated there are 2050 NHS inpatient rehabilitation beds in England. In Nottinghamshire there are two rehabilitation units ( $\mathrm{n}=37$ beds) funded by the NHS which provide inpatient rehabilitation support. Unit 1 is a high dependency unit, described as a "locked ward" by its service users and staff. It provides care to service users, aged between 18 and 65 years, who are detained under the Mental Health Act 1983 and who are experiencing enduring mental health needs and challenging behaviors. This service provides care to service users with severe and multiple comorbid needs who pose a risk to self and/or others and who present challenging behavior. Within this unit, staff support service users to reduce challenging behaviors, encourage engagement with treatment and medication, and support (re)establishing contact with support networks. This unit aims to help service users progress in their recovery so that they are able to transition to a community unit. Unit 2 is a community rehabilitation unit, informally described by staff and service users as an "open unit" which provides care to service users, aged between 18 and 65 years, experiencing enduring mental health needs and challenging behaviors. This community rehabilitation unit provides support to further recovery through psychosocial interventions, support in medication management, and encourages independent living. People using this service are often referred from high dependency units such as unit 1 as a step-down service which works towards recovery with the aim of living in supported accommodation within two years.

Quantitative Design

\section{Design and Variables}

A mixed design was used that combined a between subject's variable, Rehabilitation Service (Unit 1; Unit 2) with a within subject's variable, Time Period, (Prior rehabilitation; Post rehabilitation). To assess changes in service utilization over time, data was compared between the 3 years prior and the 3 years post a service users' rehabilitation stay.

The outcome measures used as dependent variables were; (1) the number of inpatient admissions to NHS mental health wards, (2) the number of occupied bed days in NHS mental health wards, (3) the number of Mental Health Act sections used and, (4) the amount of contact hours with NHS community mental health teams. All four outcome measures were compared prior and post rehabilitation stay and between rehabilitation services.

The outcome measures chosen were the same as those used by previous research [11-14]. Service utilization measures have previously been identified as a simple proxy of relapse and therefore a reduction in these measures would indicate a positive change following rehabilitation.

\section{Participant Selection}

Service utilization data was available for forty-seven service users (Unit 1, $\mathrm{n}=26$; Unit 2, $\mathrm{n}=21$ ) who had data for the 3 years leading up to and three years following their rehabilitation stay. These data were used in the analysis. The mean age of this sample was 37.6 years $(\mathrm{sd}=11.85)$. No personal data were available in relation to these service users' gender, ethnicity and mental health diagnosis in order to comply with General Data Protection Regulations (GDPR) and the 
sharing of personal data for research purposes. It was not possible to establish from the dataset provided for independent analyses which service users resided in the local area and those who had been admitted to the rehabilitation services from outside the area.

\section{Statistical Analysis}

The data analysis was performed using IBM SPSS Statistics (version 24). ANOVA was used to identify significant changes in the four outcome measures from prior to post rehabilitation and across rehabilitation services. Bivariate correlations were used to identify any significant relationship between time spent in rehabilitation and outcomes post discharge. All data provided was routinely collected by the NHS Trust operating the services and therefore was no missing data for any of the variables.

\section{Qualitative Design}

\section{Research Team and Reflexivity}

The research team comprised of Principal Investigator (PI), Professor of Mental Health (DB), and CoInvestigators (CI's) Research Fellow (CDM) and Research Assistant (GM). All are female and educated to doctoral level in a subject in the social sciences. The PI holds the role of Non-Executive Director within the NHS Trust operating the rehabilitation services and therefore had some knowledge of the closure of the rehabilitation beds. To avoid any bias towards the qualitative data collection and effect on participants' responses, the PI was not involved. The CI's had no conflicts of interest or direct involvement with the NHS trust and thus led the data collection and data analysis. The CI's had no previous interaction with any participant who took part, or with staff who helped facilitate the evaluation.

\section{Theoretical Framework}

The theoretical framework underpinning the qualitative part of this evaluation is phenomenology. An existential phenomenological framework allows the participants to reflect on their lived experiences and explore the significance of these experiences in relation to the topic of study [24].

\section{Participant Selection}

Four focus groups were conducted involving 22 participants, 14 service users, 6 carers and 2 staff. All service users and carers had experience of using or caring for someone who had used mental health services provided by the local NHS Trust. Due to a convenience sampling strategy it was not possible to target only service users who had specified they had been in rehabilitation services. All service users and carers who attended were aware of the closure of the rehabilitation services by the NHS Trust and the services that continued to be in place post closure. Carers who attended the focus group had no relationship with and were not caring for any of the service users who attended. The staff members who attended the focus group at Unit 1 were both nursing staff. They had each been working in rehabilitation services within the NHS Trust when the re-provision of the service has taken place.

Three of the focus groups were attended by service users and carers $(n=20)$ who were recruited using convenience sampling through two Involvement Centers run by the NHS Trust. The Involvement Centers provides service users and carers an opportunity to support each other. The focus groups were advertised to run on certain dates and all service users and carers who attended the Involvement Centers were informed about the focus groups and invited to attend.

A separate focus group was attended by staff members from Unit $1 \quad(n=2)$. Both these staff members had over 10 years of experience of working in inpatient rehabilitation services. Staff from Unit 2 were invited to take part in the focus group but declined to participate.

All focus groups were led by a member of the evaluation team (CDM and GM). Focus groups with service users were held at the Trust's Involvement Centers for the convenience of service users who were familiar with the environment and therefore more likely to feel comfortable enough to be able to share their experiences openly. The focus group with staff was held at Unit 1.

\section{Data Collection}

The topic guide for the focus group was based on the CI's (CDM and GM) presenting some preliminary findings from the quantitative data analysis for 
discussion. This was intended to stimulate discussion and provide the participants with a sense of empowerment to discuss what they felt was important. This was done initially in small groups and then if the small groups wished to share their thoughts with the rest of the group, there was an opportunity to do so. A schedule of questions designed from a recent literature review was used to inform a discussion which centered on their experience of inpatient and community mental health services and the re-provision of services in Nottinghamshire. This discussion took place as group and all participants were gently encouraged to express their experiences.

Following verbal and written consent from the participants, all focus groups were audio recorded to allow for transcription afterwards. This ensured all voices were captured to accurately reflect the opinions and perspectives of the participants. The CI's also made contextual notes that were used to enhance analysis and interpretation. The duration of each focus group ranged from 60 to $90 \mathrm{~min}$. No repeat or followfocus groups were offered as the evaluation team considered they had achieved data saturation once four focus groups were completed.

\section{Data Analysis}

Qualitative data from focus groups were audio recorded and transcribed verbatim. The transcripts were analyzed using a reflexive approach to thematic analysis which allowed multiple authors to identify patterns and themes in the data collaboratively [25]. Two members of the evaluation team (CDM and GM) followed the analysis steps suggested by Braun and Clark [25]: reading, re-reading, note taking, developing initial themes, before refining. The two evaluators allowed the themes to evolve organically and regularly discussed their interpretations of the data before agreeing a final set of common themes.

\section{Results}

Quantitative Findings

The NHS Trust provided anonymous data on service users discharged from both services between 2013 and 2018 ( $n=193$ ). Data was not used from service users who had been discharged later than 2015 as 3 years of data post discharge would not have been available at the point of data analysis (April 2018). Data was only used from service users who had experienced one stay in rehabilitation to ensure the prior and post data were distinct. Therefore, the final sample comprised 47 service users (Unit 1, $\mathrm{n}=26$; Unit 2, $\mathrm{n}=21$ ) who had all been discharged between 2013 and 2015.

\section{Admissions}

ANOVA showed a significant effect of rehabilitation on the amount of admissions to mental health wards with the number of admissions being significantly less post rehabilitation stay compared to prior rehabilitation stay $\left(F(1,45)=13.69, p<0.001, \eta p^{2}=0.23\right)$. Overall, there were significantly more inpatient admissions from service users from Unit 1 when compared with Unit $2(F(1,45)=5.49, p<0.02$ $\left.\eta p^{2}=0.11\right)$. There was no significant interaction effect which suggests the reduction in admissions was present in both rehabilitation services $\left(F(1,45)=1.74, p>0.05, \eta p^{2}=04\right)$.

\section{Occupied Bed Days}

The findings showed a significant reduction in the amount of occupied bed days on mental health wards with these being significantly less in the 3 years post rehabilitation compared to the 3 years prior rehabilitation $\left(F(1,45)=6.31, p<0.02, \eta p^{2}=0.12\right)$. There was a significant effect of rehabilitation facility with occupied bed days being greater in service users from Unit $1\left(F(1,45)=8.61, p<0.01 \eta p^{2}=0.64\right)$. There was no significant interaction effect $(F(1,45)=0.00$, $\left.p>0.05, \eta p^{2}=<0.001\right)$.

\section{Use of Mental Health Act Sections}

The use of Mental Health Act sections was found to significantly reduce post rehabilitation stay $\left(F(1,45)=6.76 p<0.02, \eta p^{2}=0.13\right)$ and the use of sections was significantly higher in service users from unit $1\left(F(1,45)=20.72, p<0.001 \eta p^{2}=0.32\right)$. There was no significant interaction meaning the reduction was not affected by the rehabilitation facility $\left(F(1,45)=2.12, p>0.05, \eta p^{2}=0.05\right)$. 


\section{Community Contact Time}

There was no significant change in the amount of contact minutes with community mental health teams prior to post rehabilitation stay $(F(1,45)=1.32$ $\left.p>0.05, \eta p^{2}=0.03\right)$. Overall community contact time was significantly higher in patients from Unit 1 compared to Unit $2\left(F(1,45)=6.95, p<0.01 \eta p^{2-}\right.$ $=0.13)$. There was no significant interaction found between the variables $F(1,45)=0.62, p>0.05$, $\eta \mathrm{p}^{2}=0.01$.

The means for each outcome measure and significance level are displayed in Table 1

\section{Time Spent in Rehabilitation and Outcomes Post Rehabilitation}

When examining the amount of time spent in rehabilitation across both services a significant correlation was found between this and the number of admissions post rehabilitation $(r(47)=-0.3, p<0.04)$ indicating that as the amount of time in rehabilitation increased the total number of inpatient admissions decreased.

A significant correlation was also found between the amount of time spent in rehabilitation and the amount of occupied bed days post rehabilitation $(r(47)=-0.34, p<0.02)$. This suggests that as the amount of time in rehabilitation increased, the number of occupied bed days decreased.

\section{Qualitative Findings}

Thematic analysis of the qualitative data demonstrated that patients, carers and staff narrate their experiences of rehabilitative recovery in accordance with three major themes, (1) encourages social integration, (2) the importance of therapeutic staff-patient relationships, and (3) rehabilitation aids personal recovery. Less pertinent, but still relevant areas of analysis are included in the presentation of the analysis (minor theme) to illustrate transparency of analysis to the reader. The minor theme presented is (4) Cost as a factor for closing rehabilitation beds. The themes are presented in Table 2.

\section{Encourages Social Integration}

Staff assert that rehabilitation makes it easier for patients to integrate back into the community. The transition from an acute setting to the community can be abrupt and rehabilitation provides an in-between step. If patients are discharged into the community without the correct support, they are likely to be readmitted. Therefore, rehabilitation gives patients the opportunity to integrate gradually back into the

Table 1 Mean number of admissions, bed days, Mental Health Act sections and community contact minutes overall for both rehabilitation facilities

\begin{tabular}{|c|c|c|c|}
\hline & 3 years prior rehabilitation stay & 3 years post rehabilitation stay & Significance level \\
\hline \multirow[t]{2}{*}{ Number of admissions } & Mean $=5.91$ & Mean $=3.15$ & \multirow[t]{2}{*}{$p<.001$} \\
\hline & $\mathrm{SD}=5.18$ & $\mathrm{SD}=3.84$ & \\
\hline \multirow[t]{2}{*}{ Number of occupied bed days } & Mean $=264.32$ & Mean $=154.38$ & \multirow[t]{2}{*}{$p<.02$} \\
\hline & $\mathrm{SD}=222.77$ & $\mathrm{SD}=224.65$ & \\
\hline \multirow[t]{2}{*}{ Number of Mental Health Act Sections } & Mean $=4.89$ & Mean $=2.83$ & \multirow[t]{2}{*}{$p<.02$} \\
\hline & $\mathrm{SD}=4.29$ & $\mathrm{SD}=3.61$ & \\
\hline \multirow[t]{2}{*}{ Community contact minutes } & Mean $=8953.55$ & Mean $=7381.89$ & \multirow[t]{2}{*}{$p>.05$} \\
\hline & $\mathrm{SD}=7212.1$ & $\mathrm{SD}=6532.85$ & \\
\hline
\end{tabular}

Table 2 Major and minor themes
Major theme

Encourages social integration

Effective staff-patient working relationship that aids recovery

Personal recovery

Minor theme
Financial incentives 
community which can then result in fewer re-admissions into inpatient services.

To me it's just a breather. That's how I describe it. You're on the acute ward you get to a level where you are not acutely unwell, and you need a breather before you learn how to integrate back into society without just being pushed and then feel like somebody's chucked you into the sea, struggling to swim. [Staff member]

They [patients] come out in the community, they find they weren't quite ready yet because they've not had that extra step-down level. And you know a lot of patients, ones had come from acute beds as well, and gone straight into the community without any chance of going through any rehab...I think that's impacted on them coming straight back and revolving in and out of care again. [Staff member]

Patients and carers suggest that social integration back in the community can be a difficult process. They voiced concerns that they can feel increased feelings of isolated in a community setting as opposed to an inpatient setting which can then lead to re-admission back into hospital.

I think coming back to this people will do whatever they can to get back into services if they're not getting the right support in the community. They'll deliberately not go to appointments, or not take their medication, or not go to have an injection. [Patient]

People can get isolated, no social interaction and that's probably even worse than being institutionalized because at least you're around people in an institution and you've got some sort of social contact. [Carer]

\section{Effective Staff-Patient Working Relationship That Aids Recovery}

Secondly, inpatient rehabilitation provides staff with the opportunity to build an effective therapeutic relationship with the patient. A key aspect of this is being able to spend a longer period with the patient. Staff acknowledged that patients can remain in rehabilitation for extended periods and that this can be preferential to the quick turnaround that is experienced on acute wards.
Erm we do like to try, and again that's one of things that we offer, we try to build a very good therapeutic relationship with our patients and work with, you know as close with them as possible erm and to a general level you know become quite friendly with some of the patients. Because some of them here can be here 15 months some have even been here a little bit longer. [Staff member]

Also, key to this relationship is the opportunity this provides for working with patients who are reluctant to engage. Rehabilitation can lead to greater success with these patients than what may be achieved if they were discharged straight into a community setting. This will then contribute to an increased likelihood of patients who are typically hard to engage remaining successfully in the community.

We can still see them every day, still chip away a little bit and slowly but surely over that 15 -month period... you know we get chance to really work hard with some people. You know increase the motivation, increase the skills and everything hopefully give them a chance. [Staff member]

This contrasts with support received in the community which was not always perceived as adequate as teams are understaffed and not able to respond in a timely manner.

I don't think they've got, they haven't got in place the time and the teams to get round to everybody. [Patient]

Yes, we've seen a lot of evidence, all of own experiences I think, of people who go out into the community and the contact they have is reduced and reduced and reduced. So, there's got to be a robust decision making on who's getting reduced contact from people, from whoever it may be. It's got to be properly done cause we've found, haven't we? That it isn't, just seems to slip for no reason. It's not, it's not as a result of a comprehensive discussion that carers or patients have been involved in. [Carer]

\section{Personal Recovery}

Thirdly, staff suggest that rehabilitation can provide patients with the opportunity to learn new skills, 
manage anxiety and gain confidence. This in turn will enable them to be better equipped for life in the community and therefore contribute to a reduction in further re-admission back into hospital. A range of activities are provided while in rehabilitation.

We have a men's group. I do er cooking with er one of the patients on that day. Erm on another day you know we have, we do gym and swim, patients go out and use the gym and go swimming. We have coffee groups, patients go out for coffee.... We have the activities day where people go out running. You know can be out gardening... Some people do their own self catering. I, basically we cover all angles really, anxiety management. Lots and lots of different things. [Staff member]

Patients and carers discuss how these elements are currently lacking from acute inpatient services and community mental health services. Patients and carers desire a greater variation of support in the community. It is also felt there should be more activities and alternative sources of support available. Social interaction and opportunities to meet with other patients and carers are an important part of recovery and this range of support is missing in the community.

There are no suitable rehab activities which help the patient's wellbeing. And when a patient comes out of hospital, we feel that it's then usually down to the family and friends to give the necessary care that they need because there's not enough contact time from the NHS. [Carer] The only thing they've ever offered [son] is a music group one night a week. Nothing else at all. [Carer]

\section{Financial Incentives}

Qualitative data from service users, carers and staff suggested the NHS trust's motivation to reduce costs money was a strong reason for the trust closing the 93 beds. Yet their experience of rehabilitation is that is an effective method of recovery, and this discuss their perspective that continuing to provide a rehabilitation service to people experiencing long-standing mental distress would reduce long-term costs.
It [rehabilitation] was, in my eyes, it always a good way of saving money because you know that revolving patient thing is one of the biggest costs to the NHS. [Staff member]

I'm not so sure that...caring for people in the community in their own homes is that much cheaper than in hospital....I think the costs involved in pushing people into their own homes when really they would be better served elsewhere and I think, I don't think the Trust has an understanding of all those costs. [Service user]

Service users and carers felt investment in or promotion of more alternative sources of support in the community would help further reduce costs.

I feel more funding should go into like drop in centers.... And then, then you could kill two birds with one stone for the want of a better word, people can go to them er and then they can be monitored er casually there. But at least they're present and they aren't going off the map and getting er perhaps ill-er and then they end up back in the hospitals which costs more money than erm the intended purpose of closing these rehabs. [Service user]

\section{Discussion}

This paper has examined inpatient rehabilitations service in one area in England following the a programme of planned bed closures in parts of the service. The findings suggest that inpatient rehabilitation in these services which existed before and after a closure programme in other parts of the service resulted in a reduction in occupied bed days in mental health inpatient services, admissions to inpatient services and use of Mental Health Act sections. The findings offer some evidence that inpatient rehabilitation services contribute to a reduction in use of mental health services and therefore improve outcomes for service users. The findings also demonstrated a correlation between the length of time spent in rehabilitation and the amount of service use post discharge, showing that the longer service users spent in rehabilitation the less admissions and bed days they experienced post discharge. These correlations offer further evidence for the usefulness of inpatient 
rehabilitation. It indicates that in this sample a greater length of time spent in rehabilitation at least initially has the potential to be linked to improved outcomes post discharge.

Qualitative data from staff with extensive experience of working in rehabilitation facilities was valuable in offering insight into these services. Staff narratives suggest that rehabilitation is effective at preparing patients for life in the community by providing vital skills for mental health recovery. This supports research that argues rehabilitation takes a more holistic approach compared to acute mental health wards which are not always able to promote independence and improve patients' social functioning [4]. Staff here spoke of how, over long periods, they can build a good therapeutic relationship with the patients which contrasts with acute wards which typically have a high turnover of patients.

The findings support previous research [11-14] which demonstrated that time in rehabilitation results in reduced readmission rates to hospital. By employing a qualitative element of the study, this research was able to explore in greater depth how inpatient rehabilitation contributes to improved outcomes in service users.

The significant correlation found between duration of inpatient admissions and occupied bed days following discharge raises important questions on the length of ideal time in an inpatient facility. This finding implies that a longer time spent in rehabilitation has the potential to produce improved outcomes post discharge. Staff narratives suggest that this is because the long-stay nature of rehabilitation care affords them the time to develop an effective therapeutic relationship with the patient. Having more time to work with patients who are reluctant to engage can lead to a better chance of a successful outcome for these individuals when discharged.

Experiencing complex and enduring mental illnesses can make recovery a difficult process with service users being repeatedly admitted to acute inpatient services [9]. This 'revolving door syndrome' places a huge demand on services, prevents other patients accessing beds, and results in high costs. For individuals with complex mental illnesses inpatient rehabilitation provides service users with the opportunity to learn new skills, develop good relationships with staff and become fully prepared for life outside of hospital.
The study has some limitations, the small sample size and all data originates from one NHS Trust in England, biases the sample and limits the generalizability of the findings to a cohort of services users and carers who experienced the bed closures. Descriptive clinical information, about diagnoses, comorbidity, severity or duration of illness were not provided. This limits the conclusions of the findings as this study is not able to suggest which group of patients benefit from rehabilitation care.

Convenience sampling was used for the focus groups recruitment which may have affected the findings. All service user and carers attended the Involvement Centers run by the NHS so were currently actively engaged and this may have biased the findings. Staff from unit 2 did not participate in any focus groups. Having their experiences absent from the analysis also highlights the limitations of the sampling as staff experiences and perspectives across both services were not captured. It was later fed-back that there was much ill-feeling regarding Nottinghamshire NHS trusts justification for closing the rehabilitation beds [26]. This resulted in a high staff turnover as well as reluctance across the remaining staff to attend a focus group to discuss their experiences further as they felt their voices and opinions had not been heard during the consultation period prior to the bed closure.

In addition, factors unrelated to the rehabilitation process may have affected the outcomes seen post rehabilitation. For example, it could be that reductions in service use post rehabilitation are partly due to differences in service provision over time due to NHS mental health services continually changing. Factors such as the different types of support available post discharge were also not examined here but are likely to have influenced the outcome measures.

Although one of the aims of the re-provisioned rehabilitation service was to ensure the right people were being admitted into inpatient rehabilitation, the study did not examine the suitability of the service users using the rehabilitation beds. Further research may want to examine this to identify what type of service user rehabilitation works best for and the alternatives routes that are taken by service users who are denied a place in inpatient rehabilitation. The findings showed that all measures of service use were significantly higher in service users from unit 1 compared to unit 2 . This reflects the 'locked' element 
of unit 1 , suggesting that service users here had more complex needs than in unit 2.

Most importantly, this paper provides evidence of specific areas of inpatient rehabilitation which future evaluations should explore when evaluating the benefits of this form of care. This paper recommends statistical comparisons pre and post inpatient rehabilitation, that specifically examine service users' number of (1) admissions, (2) occupied bed stays, (3) Mental Health Act sections and (4) community care contact time. Additionally, detailed exploration with service users, carers and staff on themes of social integration, the importance of therapeutic staff-patient relationships, and how rehabilitation aids personal recovery, could strengthen the knowledgebase of clinicians and researchers who wish to identify better care models and systems of care.

\section{Conclusion}

The findings of this evaluation suggest that inpatient rehabilitation results in a significant reduction in service use. The qualitative data highlights the unique opportunity for support that rehabilitation provides and how can be very effective for patients before they return to the community. Rehabilitation has the potential to provide the NHS with a treatment model that reduces the revolving door to inpatient care, consequently reducing costs, and giving people who are experiencing complex and enduring mental illnesses the opportunity to live successfully in the community.

The qualitative findings here are important as they highlight how a rehabilitation facility strikes the balance between restriction and freedom, social integration and isolation. The findings illustrate how a rehabilitation facility may provide effective recovery in a place of equilibrium for patients who are currently floating between inpatient and community care in their journey.

This paper offers an evaluation of inpatient rehabilitation using both quantitative and qualitative methods. Together, these findings suggest that the inpatient rehabilitation services evaluated here contributed to a reduction in service use post discharge, highlighting the continued importance of this setting within the NHS.
Author Contributions All authors contributed to the study conception and design. Statistical data preparation and analysis was performed by Gabriella Mutale. Qualitative data collection and analysis was performed by Claire de Motte and Gabriella Mutale. The first draft of the manuscript was written by Gabriella Mutale and all authors commented on subsequent versions of the manuscript. All authors read and approved the final manuscript.

Funding This study was funded by Nottinghamshire Healthcare NHS Foundation Trust (Grant No. 110083247).

\section{Compliance with Ethical Standards}

Conflict of interest Di Bailey holds the position of Non-Executive Director at Nottinghamshire Healthcare NHS Trust. Gabriella Mutale and Claire de Motte declare they have no conflict of interest.

Ethical Approval Ethical approval for the evaluation was obtained from NTU BLSS CREC. All service users, carers and staff at focus groups gave consent for their data to be used in the evaluation. All quantitative data provided by the Trust was in anonymized form and had no identifiable information relating to individual service users.

Open Access This article is licensed under a Creative Commons Attribution 4.0 International License, which permits use, sharing, adaptation, distribution and reproduction in any medium or format, as long as you give appropriate credit to the original author(s) and the source, provide a link to the Creative Commons licence, and indicate if changes were made. The images or other third party material in this article are included in the article's Creative Commons licence, unless indicated otherwise in a credit line to the material. If material is not included in the article's Creative Commons licence and your intended use is not permitted by statutory regulation or exceeds the permitted use, you will need to obtain permission directly from the copyright holder. To view a copy of this licence, visit http://creativecommons.org/licenses/by/4.0/.

\section{References}

1. Killaspy H, Harden C, Holloway F, King M. What do mental health rehabilitation services do and what are they for? A national survey in England. J Ment Health. 2005;14:157-65.

2. Killaspy H, Rambarran D, Bledin K. Mental health needs of clients of rehabilitation services: a survey in one trust. J Ment Health. 2008;17:207-18.

3. Care Quality Commission. Mental health inpatient rehabilitation services. Ward types, bed numbers and use by clinical commissioning groups and NHS trusts. 2019. https://www.CareQualityCommission.org.uk/sites/default/ files/20180301_mh_rehabilitation_briefing.pdf. Accessed 5 Nov 2019.

4. Holloway F. The forgotten need for rehabilitation in contemporary mental health services: a position statement from 
the executive committee of the Faculty of Rehabilitation and Social Psychiatry, Royal College of Psychiatrists. 2019. https://www.rcpsych.ac.uk/pdf/frankholloway_oct05.pdf. Accessed 5 Nov 2019

5. Wolfson P, Holloway F, Killaspy H. Enabling recovery for people with complex mental health needs. A template for rehabilitation services. 2019. https://www.rcpsych.ac.uk/ pdf/fr_rs_1_forwebsite.pdf. Accessed 5 Nov 2019

6. South London and Maudsley NHS Foundation Trust \& South West London and St George's NHS Mental Health Trust. Recovery is for All. Hope, agency and opportunity in psychiatry. A position statement by consultant psychiatrists. 2019. https://www.rcpsych.ac.uk/pdf/Recovery $\% 20$ is $\%$ 20for\%20all.pdf. Accessed 5 Nov 2019

7. Mountain DA, Killaspy H, Holloway F. Mental health rehabilitation services in the UK in 2007. Psychiatr Bull. 2009;33:215-8.

8. Killaspy H, Zis P. Predictors of outcomes for users of mental health rehabilitation services: a 5 years retrospective cohort in inner London, UK. Soc Psychiatry Psychiatr Epidemiol. 2013;48:1005-122.

9. Killaspy H, Marston L, Omar RZ, et al. Service quality and clinical outcomes: an example from mental health rehabilitation services in England. $\mathrm{Br} \mathrm{J}$ Psychiatry. 2013;202:28-34.

10. Bayliss P, Dhonmnacha KN, Allen D, Coe S, Ricahrds A. Evaluating the impact of inpatient mental health rehabilitation on quality of life and recovery. J Psychosoc Rehabil Ment Health. 2019;6:67-73.

11. Awara MA, Simon P, Lewis N, Edem D, Morrison JM. Psychiatric rehabilitation: quality of care and clinical effectiveness. J Psychosoc Rehabil Ment Health. 2017;4(1):61-71.

12. Bunyan M, Ganeshalingham Y, Morgan E, et al. In-patient rehabilitation: clinical outcomes and cost implications. BJPsyc Bull. 2016;40:24-8.

13. Pertie RXA, Mountain DA. An observational study of the impact of a rehabilitation admission on readmission data. Scott Med J. 2009;54:32-4.

14. Tsoutsoulis K, Maxwell A, Padinjareveettil APMT, Zivkovic F, Rogers JM. Impact of inpatient mental health rehabilitation on psychiatric readmissions: a propensity score matched case control study. J Ment Health. 2018;1-9

15. Thornton J. Locking wards has no place in psychiatric rehabilitation, says royal college. BMJ. 2018;363:k5294.

16. Pawson R, Tilley N. Realistic evaluation. 2019. https:// www.communitymatters.com.au/RE_chapter.pdf. Accessed 5 Nov 2019.

17. Bailey D, Kerlin L. Can health trainers make a difference with difficult-to-engage clients? A multisite case study. Health Promot Pract. 2016;16(5):756-64.

18. Ward J, Bailey D. How far can a short leadership and management programme address the challenges for first line social work managers? An evaluation of one of the skills for care leadership and management demonstration sites. Pract Soc Work Action. 2015;28:81-303.

19. Warr P, Bird M, Rackham N. Evaluation of management training. London: Gower Press; 1970.

20. Kirkpatrick DL. Evaluating training programs: the four levels. San Francisco: Berrett-Koehler; 1994.

21. Bailey D. Training together-part two: an exploration of the evaluation of a shared learning programme on dual diagnosis training for specialist drugs workers and Approved Social Workers (ASWs). Soc Work Educ. 2002;21:685-99.

22. Bailey D. Evaluating training for collaborative practice for graduate primary care mental health workers: part 2 . J Mental Health Train Educ Pract. 2007;2:19-29.

23. Bailey D, Littlechild R. Devising the evaluation strategy for a mental health training programme. Evaluation. 2001;7:351-68.

24. Langdridge D. Phenomenological psychology: theory, research and method. Harlow: Pearson Education; 2007.

25. Braun V, Clarke V. Using thematic analysis in psychology. Qual Res Psychol. 2006;3(2):77-101.

26. Gedling Eye. NHS chiefs to discuss Broomhill House Closure. 2015. https://www.gedlingeye.co.uk/news/nhs-chiefsto-discuss-broomhill-house-closure-plans-with-public/. Accessed 16 Jan 2020

Publisher's Note Springer Nature remains neutral with regard to jurisdictional claims in published maps and institutional affiliations. 